\title{
Relaciones intergeneracionales, demografía y economía en relación con las pensiones
}

\author{
Juan Antonio FERNÁNDEZ CORDÓN \\ Consejo Superior de Investigaciones Científicas \\ jadecala@telefonica.net
}

Recibido: 29-03-2015

Aceptado: 15-06-2015

\begin{abstract}
RESUMEN
El sostén de las personas dependientes exige una organización social que siempre está fundamentada en la reciprocidad intergeneracional. Ningún sistema de pensiones puede pretender situarse fuera de este marco. La relación nunca es consigo mismo y siempre involucra a tres generaciones. El sistema de capitalización, cuya lógica se intenta introducir en el sistema público de pensiones a golpe de reformas, hace recaer todo el riesgo sobre el pensionista-ahorrador. Lleva a un planteamiento estrechamente contable que impide explorar soluciones innovadoras a los problemas demográficos, sociales y económicos a los que se enfrentan los países desarrollados. Muchos de los vaticinios catastrofistas sobre la perspectiva de un gasto de pensiones inasumible no resisten un análisis en el que todas las variables pueden alterarse, y en especial las que determinan la distribución de la renta. La demografía está lejos de ser el único, ni el principal, condicionante del futuro de los sistemas de pensiones. La economía está muy presente por su capacidad de crear empleo, de fomentar el aumento de la productividad y de reducir las desigualdades. El envejecimiento demográfico es un cambio social que tenemos los medios de afrontar.
\end{abstract}

Palabras clave: distribución de la renta, envejecimiento, pensiones.

\section{Intergenerational relations, demography and economy in relation with pensions}

\begin{abstract}
Support of dependent persons relies on social organization, always based on intergenerational reciprocity. No pension system may be conceived without a reference to this social frame. Dependency needs can never be fulfilled by the individual him or herself and always involve a three generations scheme. Recent reforms of the "pay-as-you-go" public pension systems are progressively introducing in them the rationale of funded pension systems, where the saving-pensioner bears all risks. This leads to a narrow accountant view on pensions that prevents exploring for innovative solutions to the demographic, social and economic problems faced by the more developed countries. Most of the catastrophic predictions of the pretended unaffordable future cost of pensions may be proven wrong if all societal variables are allowed to change, especially those affecting income distribution. Demography is far from being the main, let alone the only, factor to determine the future of pensions. The economy plays a leading role thru job creation, increase in labour productivity and reduction of inequalities. Demographic ageing is a social challenge that we are in a position to overcome.
\end{abstract}

Keywords: ageing, pensions, income distribution. 


\section{REFERENCIA NORMALIZADA}

Fernández Cordón, J.A. (2015). "Relaciones intergeneracionales, demografía y economía en relación con las pensiones”. Cuadernos de Relaciones Laborales, Vol. 33, núm. 2, p. 235-258.

SUMARIO: Introducción. 1. Los intercambios entre generaciones. 2. Los determinantes demográficos de los sistemas de pensiones. 3. La economía también cuenta. 4. Conclusión. 5. Anexo técnico. 6. Bibliografía.

\section{Introducción}

Robinson Crusoe hubiera tenido que reservar físicamente para su vejez una parte de lo que pudiera producir en su isla durante sus años válidos. Algo difícil, por no decir imposible, y que, probablemente, hubiera supuesto el deterioro de buena parte de lo eventualmente almacenado. Pero si hubiese naufragado con uno o dos meses de edad, su supervivencia hubiese sido totalmente imposible. La existencia de un individuo aislado es inimaginable y por ende no puede servir de referencia para explicar comportamientos. Cada persona pasa por un período de dependencia, la infancia, hasta que es capaz de producir, etapa durante la cual aprende a conocer su entorno e integrarse en el sistema productivo del momento. Su vida acaba con una etapa, más o menos larga, de incapacidad para el trabajo, la vejez, en la que también depende de los demás. Entre estos dos extremos, los adultos producen y se reproducen. Las modalidades por las cuales las personas consiguen que una parte de lo que producen durante su vida activa sirva para satisfacer sus necesidades cuando son dependientes, siempre apoyadas en la organización social, han variado en el tiempo, en función sobre todo del nivel de producción y de las formas sociales dominantes en cada época. Todas se basan necesariamente en la colaboración de generaciones sucesivas que conviven, con edades diferentes, en un mismo momento. Los sistemas de pensiones representan una solución relativamente reciente a una parte del problema y su análisis debe realizarse en ese marco.

El debate actual sobre la viabilidad de los sistemas públicos de pensiones y las reformas que, en los países de la Unión Europea, van progresivamente alterando sus bases y su cobertura, se apoyan sobre todo en la evolución demográfica proyectada. Los planteamientos son en apariencia sencillos, aunque más bien podrían calificarse de simplistas, en la medida en que aíslan artificialmente el problema, tratado exclusivamente desde una óptica contable y actuarial. En realidad, las pensiones no son más que una pieza del engranaje que forma nuestro sistema social para hacer frente a la dependencia, en el que se imbrican inextricablemente las dimensiones demográficas y económicas, y cuya misión es contribuir a la continuidad del sistema social y productivo. Sin embargo, el análisis económico dominante, y la práctica capitalista, ignoran ampliamente la exigencia de sostenibilidad de la interacción entre sociedad y naturaleza. Los costes que aseguran la continuidad, tanto de la reproducción de las personas y de las estructuras sociales como de nuestro entorno físico y nuestros recursos naturales, no suelen ser tenidos en cuenta, o no suficientemente. Una parte del crecimiento económico, tan mal repartido además, se produce en detrimento de esa continuidad. 
Los intercambios intergeneracionales que están en la base de cualquier sistema de asunción de los dependientes poseen características constantes, cualesquiera que sean sus modalidades, aunque sus formas concretas de organización están muy condicionadas por el régimen demográfico y económico. No se plantean los mismos mecanismos en una población estacionaria de alta mortalidad, en la que también la economía es estacionaria, que en una población madura que ha alcanzado niveles altos de esperanza de vida y, sobre todo, durante los períodos de transición, de cambio o de crisis.

\section{Los intercambios entre generaciones}

El término "generación" cubre significados dispares según los ámbitos de uso (Attias-Donfut, 1988). Existe una definición estricta utilizada por la demografía, como conjunto de personas nacidas el mismo año, pero se llama generación también a cada uno de los grupos de edad definidos por su posición en el ciclo vital, que es también el ciclo de la reproducción y de la producción. La generación de los hijos, o niños, en la que se encuentran los que todavía no son ni económicamente activos ni reproductores; la generación de los adultos dedicada a la reproducción y a la producción y la generación de los mayores que han dejado de ser económicamente activos. Los límites de estas generaciones son imprecisos y cambiantes, según las circunstancias históricas. La división por edades de una población reproduce estos grupos, con fronteras que se han modificado a lo largo de la historia. Actualmente, en los países desarrollados existe una edad antes de la cual queda prohibido trabajar (16 años en España) y una edad antes de la cual no es posible jubilarse dentro del sistema público de pensiones que ha sido, hasta $2013^{1}$, de 65 años con algunas excepciones y ciertas modalidades de flexibilidad.

El consumo de los que no se encuentran capacitados para producir se apoya en intercambios entre generaciones, basados en la reciprocidad. Esta no sigue el principio de equivalencia estricta que rige en el mercado, aunque sí existe una contrapartida, al menos a largo plazo y de una importancia comparable a lo recibido. Los intercambios basados en la reciprocidad suponen la pertenencia a un grupo social al que se atribuye estabilidad temporal y requiere una cierta confianza, facilitada por la continuidad de las relaciones entre los que han recibido y los que esperan una contrapartida (Oltsen, 2004:169). Se trata de una norma y una práctica sociales claves para el funcionamiento social (IESS, 2008).

${ }^{1}$ La Ley 27/2011 de 1 de agosto prevé que la edad de jubilación para los que hayan cotizado menos de 38,5 años aumente progresivamente hasta alcanzar 67 años en 2027. Los que hayan cotizado al menos ese número de años podrán jubilarse a los 65 . 
Hay que subrayar que la reciprocidad intergeneracional no implica nunca una dación sin contrapartida. En nuestro caso es una forma de organizar la supervivencia en los períodos del ciclo vital en los que el individuo no puede valerse por sí mismo. Aunque cada individuo produzca al menos lo que necesita para consumir a lo largo de toda su vida (incluyendo la niñez y la vejez), le es imposible transferir de la edad adulta hacia su propia infancia y extremadamente difícil, en la práctica imposible, hacerlo hacia la vejez, sin contar con un entorno social y unas estructuras e instituciones adecuadas. El modelo más eficiente involucra a tres generaciones sucesivas, en una cadena sin fin. Cada generación recibe dos veces, en su infancia, de la generación anterior, la de los padres, y en su vejez, de la generación posterior, la de los hijos. Como contrapartida, cada generación contribuye también dos veces: para el sustento de los mayores de la generación anterior y para el de los niños de la generación posterior. Las tres generaciones que intervienen en los intercambios se combinan dos a dos en una relación recíproca mediante un flujo descendente de padres a hijos pequeños, cuya contrapartida es un flujo ascendente de hijos a padres mayores. El grupo familiar ha sido tradicionalmente el marco en el que se han organizado estos intercambios

Figura 1 Esquema de reciprocidad intergeneracional en la solidaridad familiar

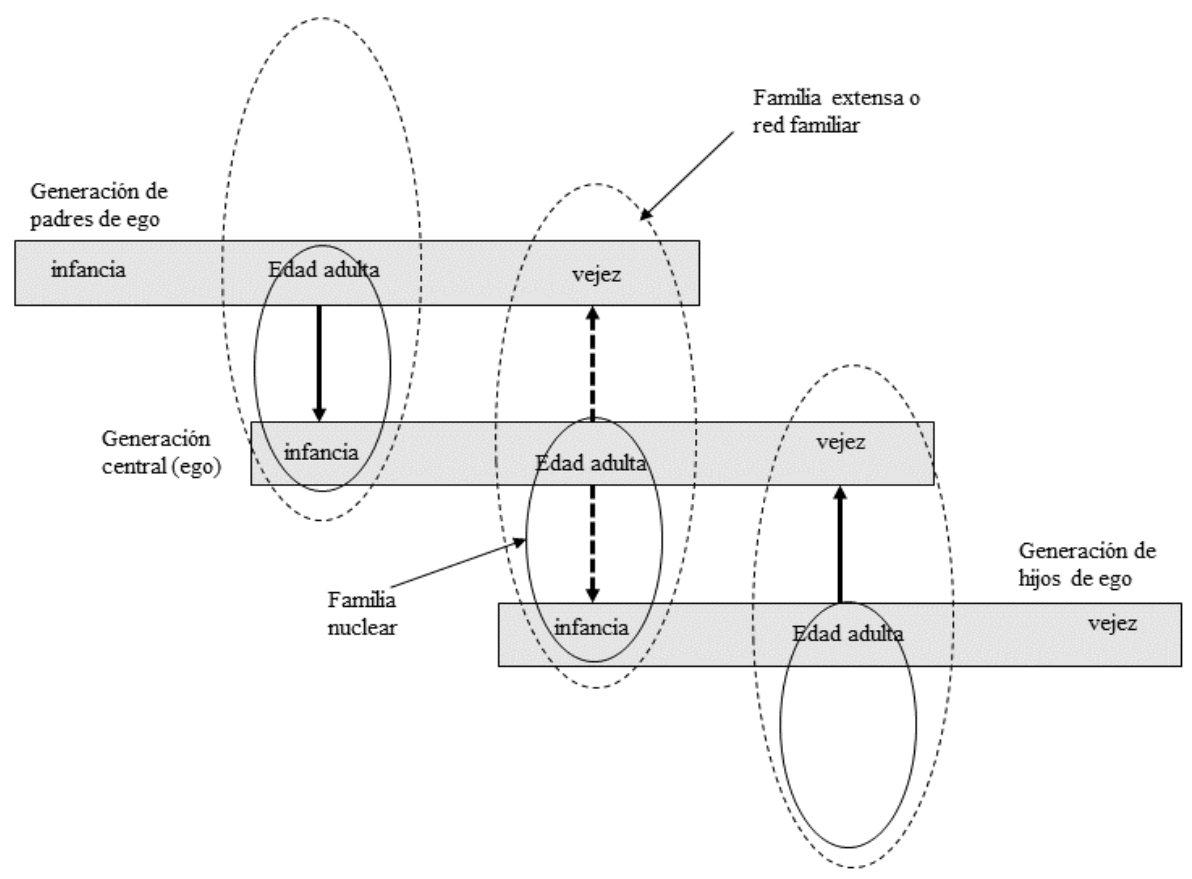


recíprocos, y su papel sigue teniendo todavía una gran importancia. La reciprocidad intergeneracional se concreta como derecho de los niños y de los mayores a una parte del producto de los adultos, en cada momento.

La imposible cobertura de los propios períodos de dependencia se resuelve, mediante el grupo familiar, estableciendo un reparto de lo que producen los adultos entre todos los miembros de la familia (Fig 1). En la larga historia de la humanidad, se organizó primero la crianza de los hijos. Sin duda porque representaban una fuerza de trabajo necesaria, aprovechable a una edad muy temprana. En las sociedades que vivían bordeando el mínimo de subsistencia, los ancianos que ya no podían trabajar no tenían derecho a seguir viviendo. Existieron sin duda costumbres y normas, aceptadas por los propios viejos, que obligaban a abandonar a los mayores cuando ya no podían participar en la producción, y simplemente mantenerlos en vida se tornaba una carga insoportable, como cuenta Jack London en su famoso cuento "Ley de vida" (London, 1901) o se ve en la "Balada de Narayama”, film de 1983 del japonés Shohei Imamura. El imperativo de mantener a los viejos solo puede surgir en sociedades con excedente, amparado generalmente en que los más ancianos conservan cierto valor para la reproducción social -la transmisión oral del conocimiento, por ejemplo- o ejercen algún poder. Puede decirse que la obligación de mantener a los mayores improductivos es una de las primeras señales de que las sociedades emprenden el camino de civilización que lleva hasta hoy. A partir de entonces, se altera la relación con la propia muerte y se inicia el largo recorrido que, apoyado en el aumento continuo de la longevidad, conduce al concepto actual de individuo y explica en gran medida los progresos materiales y sociales realizados. La transformación de la necesaria reciprocidad intergeneracional en solidaridad familiar es sin duda una de las innovaciones sociales más fundamentales. A ella están ligadas instituciones tan relevantes como el parentesco y la emergencia de relaciones afectivas o basadas en la moral, situadas fuera del ámbito económico.

La lógica económica de la protección de la vejez se fundamenta en la relación entre consumo y producción a lo largo de la vida: lo producido durante el período activo debe superar el consumo de ese período para cubrir las necesidades de los años de dependencia, además de permitir amortizar el capital utilizado en el proceso productivo. Más allá, el excedente puede dedicarse a la mejora del consumo y a la inversión en innovación e incremento de la productividad económica. A medida que ha ido aumentando la productividad del trabajo, se ha ido retrasando la edad de entrada en actividad para ofrecer un período de formación cada vez más largo, factor determinante a su vez del aumento de la productividad. También se ha adelantado la edad de cese de la actividad para permitir un cierto disfrute de tiempo libre a los trabajadores, en aceptable condición física. Al principio de la revolución industrial, el régimen salarial no difería mucho de las formas anteriores de sometimiento del trabajo (Castel, 1995:109 y sig.). Trabajaban largas horas, tanto los hombres como los niños y las mujeres. En el caso de estos últimos, las reivindicaciones obreras contaron con el interés de los capitalistas por promover una familia que fijara y estabilizara la mano de obra y por aumentar el nivel de 
conocimiento (y de adoctrinamiento) de los más jóvenes (Donzelot, 2005). Las pensiones de los viejos, por el contrario, son el resultado directo de la lucha obrera. En la invención de la jubilación pagada, que se atribuye a Bismarck, pudo más el miedo a la radicalización de los obreros que las buenas intenciones de la patronal, aunque también interesaba descartar del trabajo a los menos productivos.

Los economistas neo-clásicos han recurrido a la imposibilidad de almacenar bienes para su consumo futuro para justificar el interés del dinero y hasta la propia moneda. En esta línea de pensamiento, los intercambios intertemporales se conciben como una renuncia al consumo inmediato a cambio de una promesa de consumo futuro de mismo valor más un porcentaje (el interés) que remunera la renuncia a una postulada preferencia por el presente. El mismo bien tendrá menos valor si se sitúa en el futuro que si se dispone de él hoy. A esto se llama valor descontado a fecha de hoy de un bien futuro. En un famoso artículo de 1958, Samuelson llega a la conclusión que, en una sociedad estacionaria, un tipo de interés nulo es una situación de equilibrio que maximiza la función de consumo con relación al tiempo (Samuelson, 1958:471). Efectivamente, si la población no varía (tampoco su estructura por edades) y no hay crecimiento económico, un valor ahora es equivalente a un valor futuro. Como veremos más adelante, a propósito de las pensiones, el sistema de reparto y el sistema de capitalización son indistinguibles en ese caso.

Muchos economistas, entre ellos Kotlikoff, el padre de la contabilidad generacional, han reconocido su deuda con Samuelson. En un escrito homenaje a este gran economista, Kotlikoff retoma el modelo ultra sencillo de una sociedad de monos en una isla, formada de adultos y ancianos que se nutren de barritas de chocolate que crecen en altísimos árboles, a los que los viejos no pueden trepar. A una primera etapa en la que los jóvenes dejan morir de hambre a los viejos, le sigue el descubrimiento por estos últimos de que unas conchas pueden servir para que los jóvenes les entreguen chocolate a cambio de ellas, con el objetivo de conservarlas para, cuando llegue su vejez, intercambiarlas a su vez por chocolates con los jóvenes de entonces (Kotlikoff, 2006). ¡Los monos acaban de inventar el fondo de pensión por capitalización! Se parte, en este cuento, de una situación que ha sido real en la historia lejana, el abandono y la muerte de los ancianos por falta de alimentos, pero cuya solución fue muy distinta. Históricamente, la posibilidad de un excedente no dio lugar a la invención de la moneda, soporte de intercambios entre individuos, sino a la de instituciones sociales que creaban lazos de confianza y reciprocidad entre personas dependientes y personas productivas, como ya se ha explicado más arriba. Es significativo que los economistas neo-clásicos olviden, en muchos casos, las relaciones de intercambio no regidas por el mercado, al que parecen atribuir un carácter universal, consustancial a la naturaleza humana. Otro elemento sorprendente es que, tanto Samuelson como Kotlikoff, no incluyen en sus ejemplos didácticos, un grupo de edad inicial dependiente que debe también ser alimentado. La posibilidad de dejar morir a los niños (que también ha existido) no puede ser sistemática porque son los que perpetúan el grupo. Además, en las sociedades primitivas, representan bastante pronto una ayuda en las tareas 
productivas, aunque tarden en alcanzar el nivel de los adultos. Samuelson explica esta omisión considerando que como niños forman parte del consumo de los padres (Samuelson, 1958:468). No menciona en ningún momento que los adultos con los que tratarán los viejos son estos mismos hijos, cuyo consumo inicial han asumido ellos como padres.

Creemos que el esquema de las tres generaciones que se solapan y establecen intercambios recíprocos dos a dos, es el que mejor y más adecuadamente describe la base sobre la que se apoya cualquier sistema de sostén de los dependientes. Ha sido en el seno de la familia donde se han concretado los intercambios, mediante lo que se llama solidaridad familiar (Tobío, 2012,). Esta puede ejercerse en el ámbito amplio de una comunidad (tribu, parroquia, barrio) con cierto desbordamiento de la familia estricta, o mediante la convivencia en el seno del hogar, cuya definición estadística está precisamente basada en la puesta en común de recursos, o, como sucede actualmente, a través de redes familiares en las que se organiza la ayuda mutua entre familiares que no conviven (Tobío y Fernández Cordón, 2013).

La puesta en marcha de un sistema de pensiones, destinado a asegurar la suficiencia económica de los que ya no trabajan, aísla uno de los elementos de la reciprocidad intergeneracional, sin cambiar la base sobre la que se apoya. Históricamente, los sistemas de pensiones, implican el pago de unas cotizaciones por parte de los trabajadores, que da derecho a la percepción de una prestación periódica durante el período de jubilación. El consumo de los jubilados se detrae necesariamente de lo producido por los activos que les son contemporáneos. El sistema puede concebirse como una cadena en la que, en cada momento, los activos sostienen a los jubilados, como ocurría en la familia, o como forma de constituir una reserva que los trabajadores podrán utilizar cuando lleguen a la jubilación. En el primer caso se habla de sistema de reparto $y$, en su estado más puro, las cotizaciones simplemente prueban que la persona que se jubila aportó en el pasado su contribución y ahora puede ejercer su derecho a una pensión. Es un sistema basado en la pertenencia a un conjunto social, que gestiona y garantiza los derechos y las obligaciones de cada uno y, en última instancia, está supeditado a la suficiencia económica. El segundo caso pasa por la compra de activos financieros (las famosas conchas de Kotlikoff) por los trabajadores, que podrán vender cuando se jubilen para obtener ingresos. En los dos casos, el consumo de los mayores de una parte del producto se basa en un derecho: el que se deriva de la reciprocidad intergeneracional, en el caso del sistema de reparto, y el que protege al acreedor en los mercados.

El debate en torno a las bondades respectivas de estos dos sistemas ha sido largo e intenso, aunque actualmente sus términos se están modificando y suavizando. Nuestra tesis es que no existe entre ellos diferencias de fondo importantes. Son indistinguibles en una situación estacionaria y también cuando el sistema de capitalización llega a su madurez y funciona como si, en cada momento, los nuevos ahorradores pagasen las pensiones de los jubilados. Es frecuente que se presente como más garantista un sistema que se apoya en la acumulación de un capital (“funded pension systems” en inglés) pero, en la práctica, han resultado ser los más 
nocivos para los ahorradores, expuestos a los vaivenes del mercado y de la coyuntura económica. También es frecuente que se considere que el sistema de reparto está siempre al borde del déficit, a pesar de lo cual es, hasta ahora, el que mejor ha resistido los cambios demográficos y económicos. El vocabulario, casi nunca neutro, erige, en la literatura anglo-sajona actual, como referente al sistema de capitalización y designa en negativo ("unfunded pension systems") a los sistemas de reparto, los más extendidos.

Durante unos años, en torno a mediados de los noventa, en España se debatió la sustitución del existente sistema de reparto por un sistema de capitalización, argumentando la ruina inminente del sistema público. El acento se ponía sobre todo en los costes de la transición de un sistema a otro. El vaticinio no se ha cumplido y, actualmente, la idea de substitución ha prácticamente desaparecido del debate. Los ciudadanos, en su inmensa mayoría, confían en el sistema público de pensiones, ahora más que nunca por los efectos negativos que la crisis financiera ha tenido sobre los fondos privados. Sin embargo, esta opinión mayoritaria no parece haber afectado las preferencias del sistema financiero y de muchos economistas influyentes que siguen orientadas a una solución mixta. Por un lado, un sistema de reparto, público y obligatorio, reducido y con bajas tasas de sustitución, dotado de un fuerte componente contributivo (apto así para ser eventualmente gestionado por la empresa privada), acompañado de un sistema de capitalización voluntario y, en la práctica, vedado a los que disponen de menos recursos y despreciados por los que profesan una "alta preferencia por el presente", aunque en el futuro necesitarán una pensión, como los demás.

\section{Los determinantes demográficos de los sistemas de pensiones}

Un conocido economista, miembro del Grupo de Expertos que contribuyó al diseño de la última reforma del sistema de Pensiones, resume en un libro reciente el razonamiento demográfico que lleva a proponer recortes en las pensiones, a lo que cada reforma conduce inevitablemente. Recuerda que, cuando se crean los sistemas de pensiones, solo el 35\% de la población alcanza los 65 años, edad a la que hoy sobrevive más del $90 \%$ y que, en términos de supervivencia, 65 años de antes equivalen a 89 años de ahora, edad a la que llega actualmente el $35 \%$ de la población (Conde-Ruiz, 2014:11). De ahí la pregunta, que él contesta negativamente, “¿será posible mantener, con los actuales sistemas de pensiones públicas, un nivel de consumo suficiente para un número de jubilados, cada vez mayor?” (Conde-Ruiz, 2014:12). Un planteamiento en apariencia sencillo, con un argumento que parece irrebatible aunque, en realidad, como veremos, es limitado y parcial.

Para analizar cómo se inscriben los sistemas de pensiones en el marco demográfico, conviene apoyarse sobre el modelo estacionario, que es también lo que hace, tal vez sin saberlo, el autor citado. La estructura por sexo y edad de una población estacionaria viene totalmente determinada por una tabla de mortalidad, a 
la que la población está asociada, y que se supone constante en el tiempo. Así, a cada nivel de mortalidad corresponde una estructura poblacional, mientras el número de nacimientos anuales está ligado al volumen de la población. Comparar los casos hipotéticos de dos niveles de mortalidad muy contrastados, el de la España de principios del siglo XX (esperanza de vida al nacer de 40 años para los hombres en 1908) y el de la España actual (80 años para los hombres en 2014) permite establecer el juego de determinantes que inciden en cualquier sistema de pensiones y como les afecta el nivel de la mortalidad. En la historia concreta, intervienen los efectos de la transición que lleva de una situación a otra, por disminución de la mortalidad y posterior adaptación de los niveles de fecundidad.

Los sistemas de capitalización tienen como marco de referencia el ciclo vital de los individuos. Desde un punto de vista estrictamente demográfico, es importante para estos sistemas la relación entre los años vividos como productor y los años vividos como dependiente. Preparar la jubilación consiste, en este marco, en ahorrar durante el período productivo (comprando activos financieros, por ejemplo) y, a partir del momento de la jubilación, dedicar ese ahorro al consumo (vendiendo los activos financieros anteriormente adquiridos), generalmente en forma de renta, ya sea financiera (una cantidad mensual hasta agotamiento del fondo y su rentabilidad eventual) o asegurada (una cantidad mensual vitalicia que depende del capital y los intereses acumulados y de la edad y sexo de la persona).

Para determinar el ahorro que asegura un consumo dado a lo largo del período de jubilación (o, inversamente, calcular el nivel de consumo que permite un determinado ahorro), la variable demográfica relevante es la relación entre años en situación de jubilado y años en el sistema productivo. Esta relación, calculada en un grupo generacional, por ejemplo, depende exclusivamente de la mortalidad.

En los sistemas de reparto, la variable demográfica significativa es la relación entre la población en edad de jubilación y la población en edad de producir, en cada momento. Si se aplica estrictamente el principio de reparto (lo que no sucede en la práctica) la relación citada fija la cotización si se conoce el importe de la pensión, o fija la pensión si la cotización viene dada. El sistema tiene una lógica transversal. La población de un año dado está formada de aproximadamente un centenar de generaciones, desde la que acaba de nacer hasta la que está a punto de desaparecer. Esta relación, para un año dado, depende por tanto de los niveles de mortalidad anteriores de todas las generaciones y del número de nacimientos en cada generación, es decir de los aproximadamente cien años anteriores, no todos con la misma importancia.

La división ternaria del ciclo de vida en infancia, edad adulta productiva y vejez, se refleja en los grupos de edad correspondientes en la población. Si queremos comparar la situación de dos generaciones, desde el punto de vista demográfico, debemos relacionar los años que, en promedio, cada generación vive en cada etapa, es decir en cada grupo de edad. Por otra parte, comparar la situación de dos años de calendario supone relacionar, en cada uno, la población en edad de jubilación y la población en edad de trabajar. En una situación estacionaria, los dos objetivos se confunden. El número de años vividos en cada etapa por una generación es igual al 
efectivo del grupo de edad correspondiente en la población estacionaria, en un año cualquiera. En una situación estacionaria, el sistema de reparto y el sistema de capitalización son estrictamente equivalentes, desde el punto de vista demográfico.

La Ratio Demográfica de Dependencia por Vejez (RDDV) es un indicador fundamental que mide, en una generación, el tiempo vivido en edad de jubilación (65 o más, por ejemplo) por cada año vivido en edad de trabajar (15-64),

$R D D V=\frac{l_{65} e_{65}}{l_{15} e_{15}-l_{65} e_{65}}$

En el numerador de RDDV figuran los años vividos por una generación después de los 65 años y en el denominador los años vividos en edad de trabajar. En la población de un año dado, se puede expresar un indicador equivalente, la Ratio Demográfica de Dependencia de Mayores (RDDM) en términos de efectivos de cada grupo de edad:

$$
R D D M=\frac{P_{65+}}{P_{15-64}} \text {. }
$$

Con frecuencia la RDDM es llamada, simplemente, y muy impropiamente, ratio de dependencia. Cuando la población es estacionaria, las dos ratios son iguales, cuando no lo es (caso habitual), la estructura por edades se ve también afectada por las variaciones del número de nacimientos anteriores, y RDDM se desvía de su equivalente en la generación RDDV. En principio, la variación de los nacimientos no afecta a los sistemas por capitalización (aunque esta afirmación debe ser matizada por el efecto que los tamaños relativos de las cohortes podrían tener sobre los mercados financieros).

Los cambios que se han producido en la mortalidad desde principios del siglo XX han provocado un aumento sensible de la RDDV, de 13\% en 1908 a 38,9\% en 2014. Los años vividos a partir de 65 (efectivos de 65+ en la población estacionaria) se han multiplicado por 5.1, aunque la reducción de la mortalidad ha tenido también un efecto positivo sobre la capacidad productiva de la población, debido a la mejora de la mortalidad anterior a 65 años. Los años en el período activo se han multiplicado por 1,8. El resultado es que se ha triplicado el tiempo vivido a partir de 65 años, por cada año vivido en edad de trabajar. Las ganancias de mortalidad antes de 65 años están hoy casi totalmente agotadas y su incidencia futura puede ser considerada prácticamente nula. El elemento que tendrá un impacto apreciable es el previsible aumento de la esperanza de vida a los 65 años, único factor demográfico con incidencia en la relación fundamental que rige los sistemas de pensiones, ya sean por capitalización o de reparto. Salvo sorpresa de la medicina, el efecto del factor demográfico será en el futuro menor de lo que ha sido en el siglo pasado. Nadie espera que, en menos de un siglo, se duplique la esperanza de vida actual, llegando a los 160 años. En cuanto a la fecundidad, se encuentra, en España, por debajo del nivel de reemplazo desde el inicio de los ochenta, pero esto no ha tenido hasta ahora ninguna incidencia apreciable sobre el mercado de trabajo, ni sobre el equilibrio del sistema de pensiones, porque la inmigración ha compensado el déficit 
de nacimientos. Las proyecciones más recientes prevén un nivel de fecundidad por debajo de dos hijos por mujer y un cierto aporte migratorio que, en conjunto, equivalen a una situación de reemplazo generacional ${ }^{2}$. En resumen, el efecto demográfico adverso para los sistemas de pensiones será, en el futuro, algo inferior al observado en el siglo pasado. Las proyecciones del INE referidas a 2014, postulan un aumento de la esperanza de vida a los 65 años entre 2014 y 2063 de 8 años aproximadamente, de 21 a 29 años $^{3}$, un promedio de 2 meses más de vida cada año (INE, 2014). Así, la RDDV alcanzaría el 56,2\%, en 2063, un aumento de $44 \%$ en 50 años, cuando en los cincuenta años anteriores se incrementó en más del $60 \%$. Con una hipótesis menos optimista (y tal vez más probable) sobre la longevidad futura, el peso de la demografía sería aún menor (Cuadro 1 y Gráfico 1).

\section{Cuadro 1 Indicadores de mortalidad y de estructura de la población española 1908-2012 y proyección 2014-2063}

\begin{tabular}{|r|rr|rr|rr|}
\hline \multicolumn{1}{|c|}{ Año } & \multicolumn{2}{|c|}{ Probabilidad de supervivencia } & \multicolumn{2}{|c|}{ Esperanza de vida } & & \\
& a 15 años & a 65 años & entre 15 y 65 & a 65 años & RDDV & RDDM \\
\hline 1908 & $67,3 \%$ & $33,5 \%$ & 27,02 & 10,55 & $13,1 \%$ & $9,7 \%$ \\
1975 & $97,5 \%$ & $79,8 \%$ & 46,63 & 15,25 & $26,1 \%$ & $16,5 \%$ \\
1995 & $99,2 \%$ & $85,3 \%$ & 47,70 & 18,28 & $32,7 \%$ & $22,1 \%$ \\
2005 & $99,4 \%$ & $88,3 \%$ & 48,34 & 19,32 & $35,2 \%$ & $24,1 \%$ \\
2012 & $99,6 \%$ & $90,1 \%$ & 48,75 & 20,56 & $38,0 \%$ & $25,7 \%$ \\
2020 & $99,7 \%$ & $91,7 \%$ & 49,04 & 22,08 & $41,3 \%$ & $30,7 \%$ \\
2040 & $99,9 \%$ & $94,3 \%$ & 49,47 & 25,55 & $48,7 \%$ & $56,6 \%$ \\
2060 & $99,9 \%$ & $95,9 \%$ & 49,67 & 28,62 & $55,3 \%$ & $75,0 \%$ \\
2063 & $99,9 \%$ & $96,1 \%$ & 49,69 & 29,03 & $56,2 \%$ & $74,7 \%$ \\
\hline
\end{tabular}

Fuente: Human Mortality Database (HMDB, 2014) para el período 1908-2012; INE(2014) para las proyecciones 2014-2063

El factor demográfico de los últimos cincuenta años ha sido en parte amortiguado por nuestro sistema de reparto. Durante todo el siglo XX y, según las proyecciones del INE de 2014, hasta el año 2034, la RDDM, calculada con las poblaciones del año, es sistemáticamente inferior a la RDDV, calculada con la tabla de mortalidad del año. La transición demográfica, vivida en España a partir de principios del pasado siglo, con un cierto retraso en relación con países de nuestro entorno, se inicia con la disminución de la mortalidad en las edades más jóvenes, que va progresivamente extendiéndose hasta las más mayores. Después de un cierto

${ }^{2}$ La equivalencia no es estricta: la composición por edades de los inmigrantes no coincide con los huecos generacionales de la baja fecundidad.

${ }^{3}$ Estimación propia a partir de los datos para hombres y mujeres publicados por el INE. 


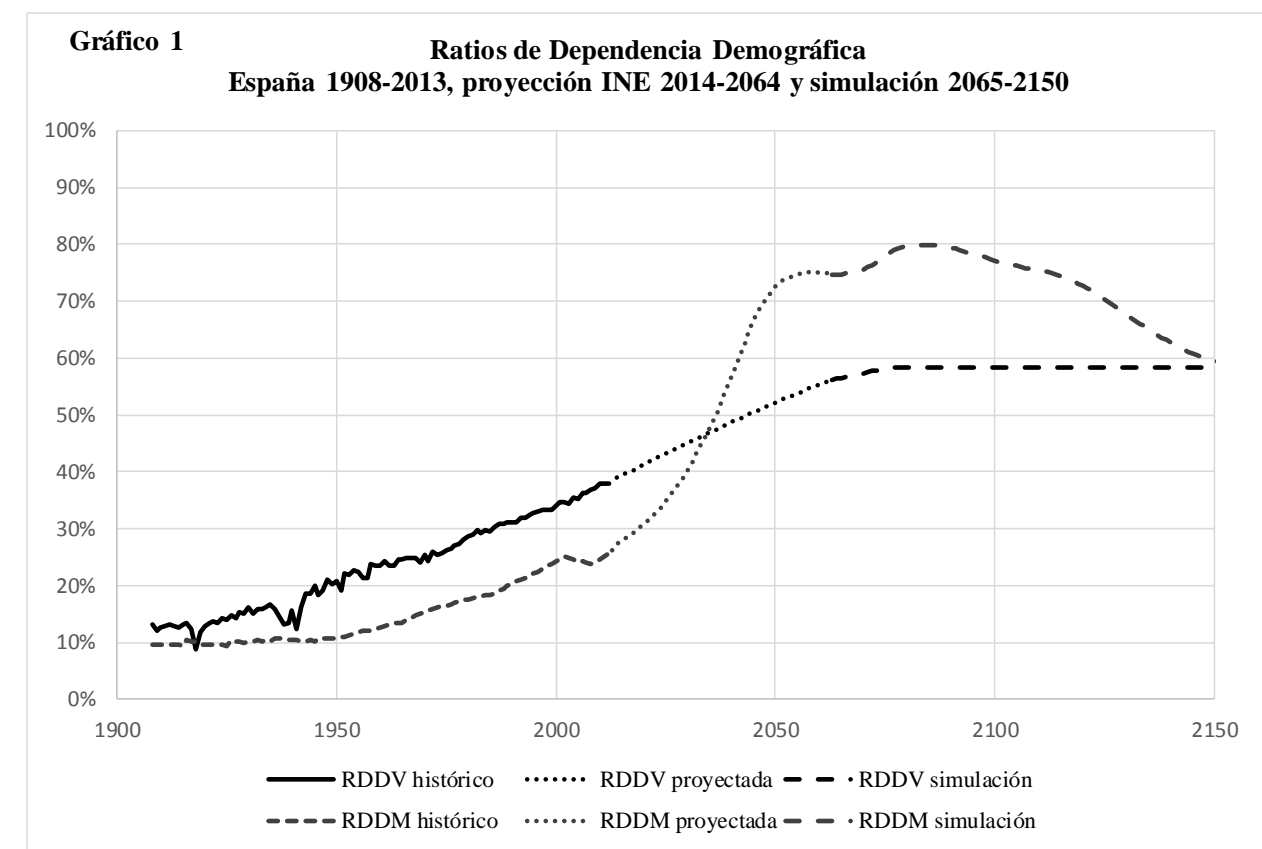

RDDV: años vividos en 65+ por año vivido entre 15 y 65, población estacionaria asociada a la tabla de mortalidad del año

RDDM: ratio de la población de 65+ a la población de 15-64 del año

Fuente: Human Mortality Database (HMDB, 2014) para el período 1908-2012;

INE(2014) para las proyecciones2014-2064; elaboración propia para la simulación 2065-2150 con mortalidad constante.

tiempo, disminuye también la fecundidad. El desfase temporal entre estos dos movimientos provoca un aumento de la población y mantiene una estructura por edades más joven. Cuando empieza a disminuir la fecundidad, se inicia un período en el que disminuye la carga de hijos y en el que aumenta el número de los que acceden al mercado de trabajo, por la menor mortalidad de los jóvenes. Al mismo tiempo, las generaciones más antiguas, que no se han beneficiado de la mayor longevidad, pierden peso relativo. Esta circunstancia da lugar a lo que varios autores han llamado "dividendo demográfico": una situación propicia al desarrollo económico (Bloom et al., 2003, Mason, 2006,). Su efecto puede dar cuenta de hasta un tercio del "milagro económico" que protagonizaron los países del Asia Oriental y también explicaría buena parte del fulgurante despegue del "Tigre irlandés" (Bloom et al. 2007:52-53). Los autores citados insisten en que para dar sus frutos, las circunstancias demográficas necesitan ser aprovechadas por políticas adecuadas que favorezcan la mejora del capital humano y la actividad orientada a la exportación. Puede añadirse que interesa, por el lado de la demanda, que los salarios, con alta propensión al consumo, no se vean gravados por cotizaciones elevadas para pensiones y, por el lado de la oferta, que los costes salariales no 
incluyan cotizaciones demasiado elevadas, para fomentar la competitividad. Es precisamente esto lo que ha permitido el sistema de reparto. Las cotizaciones, para un nivel de pensión dado, hubieran sido netamente más elevadas en un sistema por capitalización, frenando el efecto positivo del factor demográfico. Pero, hay que resaltar, que las variaciones de la estructura por edades que favorecen el crecimiento, no son permanentes. La población llegará, en su momento, a una situación de equilibrio próxima a la población estacionaria, más envejecida, y antes pasará por otra fase de mayor envejecimiento todavía que el que corresponde al equilibrio estacionario. Por ejemplo, según la citada proyección de población del INE, a partir de 2035, la RDDV correspondiente a la tabla de mortalidad de ese año es inferior a la RDDM calculada con las poblaciones del año. De alguna manera, esta situación, desfavorable al crecimiento, habrá sido previamente compensada por el dividendo demográfico. Lo deseable sería que se anticipen estos movimientos y se prevean, con tiempo suficiente, los mecanismos que hagan posible afrontar los efectos cíclicos asociados al envejecimiento demográfico. Es necesario reconocer que la aportación de los activos en los tiempos de población joven, no se limita estrictamente a su cotización. Una parte del crecimiento económico se debe a que las pensiones fueron entonces baratas. Los pensionistas que ahora llegan a la edad de retirarse pueden ejercer, legítimamente, un cierto derecho sobre la riqueza creada, lo que exigirá implantar nuevos mecanismos de redistribución. Ello no significa necesariamente que tengan que aumentar las cotizaciones ya que existen otras formas de repartir la carga, por ejemplo mediante el sistema impositivo. Todo lo contrario opinan los que creen llegado el momento de resucitar la referencia al modelo de capitalización y aplicar conceptos propios de estos sistemas, como la tasa interna de rentabilidad, al sistema público por reparto (ver, como ejemplo, Jimeno y Licandro, 1999) que llevan, inexorablemente a proponer recortes en las pensiones. Esto carece de sentido, debido a la imbricación de los sistemas de reparto en la economía, lo que no ocurre con los de capitalización. En estos últimos, la rentabilidad del capital acumulado es una variable clave (y casi la única) porque el sistema de capitalización no es más que una operación financiera de ahorro y gasto pospuesto. Maximizar la rentabilidad puede conducir a invertir en mercados extranjeros, por ejemplo, con efecto negativo para la economía del país. Impera en ellos, en todo caso, una lógica contable, que no permite modular ninguna respuesta adaptativa a los problemas que plantea la reciprocidad intergeneracional ni a los cambios sociales de envergadura. Valga un ejemplo: hemos llegado a un momento en el que parece que debe ser afrontado el coste del alargamiento de la vida después de los 65 años (a pesar de que hace un siglo que la longevidad aumenta, incluso a mayor ritmo que ahora). Trabajar más porque vivimos más es únicamente una idea entre muchas otras, pero es la única posible cuando el referente es el sistema de capitalización. Además, es de las ideas peores si nos atenemos a las dificultades que muestra el sistema para crear empleo y se tienen en cuenta las muy bajas tasas de ocupación entre las personas de más de 55 años. Nada, en el mercado de trabajo, apunta a la necesidad de retrasar la edad de la jubilación, cuya única finalidad sería reducir el gasto dedicado a pensiones, sin que el mayor número de activos implique 
un mayor número de ocupados. También es de las ideas más injustas, debido a las grandes diferencias de mortalidad entre categorías socioeconómicas, muy elevadas y sin tendencia a estrecharse (Waldron, 2009; Shkolnikov et al., 2012). Una reducción del tiempo de jubilación igual en valor absoluto para todos significa un mayor sacrificio por parte de los que menos expectativa de vida tienen, que son los menos cualificados, los menos instruidos, los que cobran las pensiones más bajas. Retrasar la edad de jubilación equivale a una redistribución de la renta de los más pobres hacia los más ricos.

La última reforma de las pensiones, recogida en la Ley 23/2013, de 23 de diciembre, contribuye a aislar aún más el sistema público de pensiones del entorno social y económico, precisamente en el momento en que ese aislamiento resulta más desfavorable para los trabajadores que han cotizado. Esta reforma ha ido más allá de lo que la de 2010 entendía por factor de sostenibilidad, un correctivo para contrarrestar el efecto del aumento esperado de la esperanza de vida. Se ha introducido además un "índice de revalorización” que, con independencia de su ropaje matemático, consiste en una fórmula para limitar el gasto de pensiones, ligándolo a los ingresos de forma inflexible (o muy poco flexible). En consecuencia, las personas que cotizaron durante el período del dividendo demográfico perderán sus derechos sobre el crecimiento económico, mediante la necesaria redistribución de la renta. Se trata de una medida tramposa, que no sirve para cualquier coyuntura demográfica ${ }^{4}$, cuya única finalidad es rebajar las pensiones. Se aplica la lógica de un sistema privado cerrado en el que el pensionista es el único que carga con cualquier tipo de riesgo.

El sistema de reparto permite instrumentar cualquier decisión, lo que no puede el sistema de capitalización, que hace necesariamente recaer sobre el ahorrador el coste de su mayor longevidad. Este solo puede optar entre cobrar menos pensión o retrasar su jubilación, dos opciones actuarialmente equivalentes. Los planteamientos hoy prevalentes, que conducen a reformar el sistema público para acercarlo lo más posible a un sistema privado, con mayor dosis de contributividad, impiden que se abra un debate real sobre los tiempos que rodean y prolongan el final de la vida laboral. La sociedad debe debatir y decidir si una parte de la riqueza creada, o de su aumento, se puede dedicar a financiar más tiempo de jubilación o, como se ha propuesto, promover una nueva "política de las edades" que adapte nuestro actual ciclo ternario de formación, actividad y ocio, a la vez a la mayor longevidad y también a los cambios en el modelo productivo y a las nuevas necesidades de formación (Guillemard, 2007). En términos generales, el sistema de reparto, está dotado de una gran flexibilidad para poner en práctica la reciprocidad entre generaciones, adaptándola a los cambios sociales y económicos, como lo

${ }^{4}$ Cuando la RRDV es superior a la RDDM (situación que se ha dado continuadamente en el pasado reciente) la lógica del índice de revalorización conduce teóricamente a inflar las pensiones del momento, en detrimento del futuro. 
viene haciendo desde hace más de un siglo. Exige, sin duda, una gestión adaptada al largo plazo, para hacer frente a las variaciones demográficas, cuyo ciclo temporal es largo, y a la coyuntura económica, en particular su reflejo en los niveles de empleo.

En resumen, los factores demográficos ejercen una influencia sobre todos los sistemas de pensiones, a través de la continua (hasta ahora) disminución de la mortalidad. En los próximos años, la variable más importante será la evolución de la esperanza de vida a los 65 años, único factor demográfico que condiciona al sistema de capitalización, pero que también debe afrontar el sistema público de pensiones. Al efecto de la mortalidad, se añade, en el caso de los sistemas de reparto, la variación de los nacimientos anuales (y eventualmente de la inmigración) con ciclos muy largos. En el pasado, el menor número de pensionistas ha favorecido el crecimiento económico. Cuando los trabajadores que han protagonizado nuestro boom económico llegan a la jubilación es necesaria una política de redistribución a su favor de la riqueza creada. En su lugar, solo prosperan propuestas de recorte de pensiones, basadas en la previsión de un déficit financiero del sistema, como si este estuviera totalmente aislado del resto de la realidad económica y social.

\section{La economía también cuenta}

La demografía es importante para las pensiones. Tan importante que constituye la base misma de cualquier sistema de pensiones. Se puede y se debe analizar la influencia de su evolución en cualquier dispositivo de atención a la dependencia. Es, además, buena práctica de un análisis riguroso aislar los componentes de un sistema para determinar el lugar que ocupan y la importancia que ostentan. Lo que no es legítimo es que ese análisis "ceteris paribus" conduzca directamente a reformas que afectan, a veces dramáticamente, la vida misma de las personas. No es suficiente para concluir que la evolución demográfica va a arruinar el sistema público de pensiones, ni que es necesario trabajar más tiempo porque vivimos más tiempo. Existen otros factores que también inciden sobre el equilibrio financiero del sistema y conviene tener en cuenta, además, las interrelaciones entre todos los factores.

Las reformas propuestas y las que ya se han realizado, se apoyan en proyecciones demográficas que cuantifican supuestos de evolución futura de los componentes de la dinámica poblacional (mortalidad, fecundidad y movimientos migratorios) basados sobre todo en tendencias pasadas y sin ninguna conexión explícita con la evolución de la economía y en particular del mercado de trabajo. Todas las realizadas recientemente anticipan un aumento del peso de mayores y una disminución de la población en edad de trabajar, que se traduce por un aumento del indicador que hemos llamado RDDM

$\frac{P_{65+}}{P_{15-64}}$ 
La primera observación es que la relación entre el número efectivo de pensionistas y el de cotizantes no sigue necesariamente la evolución de la ratio demográfica, que únicamente pone en relación grupos de edades. Mientras que parece razonable admitir una correlación alta entre el número de pensionistas y la población mayor de 65 años (o, en el futuro, de 67 años) no ocurre lo mismo con la población en edad de trabajar. En el grupo de 15-64 años, no todas las personas se encuentran efectivamente ocupadas, y por tanto en situación de cotizantes. Algunas siguen en el sistema educativo, otras están en el paro y otras figuran como inactivas (fuera del mercado de trabajo) por razones diversas: parados desanimados, amas de casa, pensionistas o jubilados anticipadamente y todas aquellas que no desean trabajar y se lo pueden permitir. La tasa de empleo global (porcentaje de las personas en edad de trabajar que ocupan efectivamente un empleo) varía también en función de la demanda de trabajo (el número de puestos de trabajo disponibles) condicionada a su vez por la situación de la economía. Por todas estas razones, la proporción de ocupados en el seno de la población en edad de trabajar es variable en el tiempo y en el espacio. En las últimas décadas, la tendencia en los países desarrollados ha sido de ligera disminución de la tasa de empleo de los hombres y de incremento de la de mujeres, muy elevado en algunos países como España, con resultado de aumento de la proporción de ocupados en la población.

La disminución de la población en edad de trabajar no representa necesariamente un obstáculo al crecimiento económico, ni siquiera si la tasa de empleo alcanzara un máximo (lo que se antoja una hipótesis lejana en la España actual). Existe la posibilidad de que lleguen inmigrantes si el sistema productivo es capaz de generar una demanda de trabajo que permita el crecimiento. Por estas razones, las proyecciones demográficas no pueden seguir tratando la demografía como variable exógena a la economía. En unos trabajos recientes hemos propuesto un modelo de proyección de la población que integra la evolución prevista del mercado de trabajo, en función de hipótesis sobre el crecimiento futuro del PIB y la evolución de la productividad (Fernández Cordón y Planelles, 2014). Los resultados muestran que las proyecciones realizadas por Eurostat para los países miembros no son, en muchos casos, compatibles con los escenarios económicos a largo plazo de la OCDE, porque subestiman la necesidad de inmigrantes, y por tanto también la población futura en edad de trabajar, aunque se produzca un aumento sensible de la tasa de empleo. De ello se deriva que las ratios futuras de dependientes por persona ocupada están siendo actualmente sobreestimadas.

La transición demográfica que, como hemos visto, no solo ha provocado un aumento de la población de mayores sino que ha mejorado la capacidad productiva, al eliminar muertes inútiles de personas jóvenes, incluye también una disminución de la fecundidad mínima necesaria para la continuidad demográfica (de cinco hijos por mujer a principios del siglo XX a dos actualmente). Este cambio ha contribuido, junto a otros, a que las mujeres hayan podido entrar y permanecer en el mercado de trabajo, aumentando considerablemente la capacidad productiva de la población. El envejecimiento demográfico, fruto de la transición, genera en cierto modo su propio antídoto. Aumenta la población dependiente de mayores pero a la vez aumenta la 
población capaz de producir y disminuye el número de menores y de mujeres dependientes.

Desde 1985 hasta la llegada de la crisis, España ha vivido un período de gran creación de riqueza, impulsada en buena parte por el aumento de la participación femenina en el mercado de trabajo. Una parte sustancial de este crecimiento ha ido a parar a los no asalariados, como indica la disminución del peso de los salarios en el PIB durante esos años. El aumento de las familias con dos salarios ha generado cambios en los modos de consumo, en los precios y en los salarios que han acabado imponiendo como norma la necesidad de dos sueldos para vivir, creando, de paso, nuevas desigualdades entre los que no cumplen con el nuevo requisito. Hoy, esta redistribución regresiva de la renta, en detrimento de los asalariados, se toma como dada y no se cuestiona a la hora de atender las necesidades del sistema de pensiones.

El sistema público de pensiones interpela al conjunto de la economía y, existiendo recursos suficientes, el problema es encontrar mecanismos adecuados que aseguren una distribución de la riqueza más justa y más acorde con las expectativas y derechos de los trabajadores ya jubilados o que se irán jubilando en el futuro. Reducir el consumo de los jubilados es ahondar aún más en la desigual distribución de la renta.

El porcentaje del PIB que va a los mayores puede aumentar en los próximos años, sin que este incremento afecte los niveles relativos de renta. La proporción del PIB que va a los mayores en un año cualquiera $\mathrm{t}, \delta_{m, t}$ se puede expresar en la función siguiente de dos variables de distribución de la renta y de la proporción de 65 años o más en la población (ver Anexo técnico).

$$
\delta_{m, t}=\frac{v_{t} \alpha_{t}}{v_{t}+\frac{1-R M_{t}}{R M_{t}}}
$$

En el ejemplo desarrollado en el Cuadro 2 del Anexo técnico, se ha utilizado la evolución de la población entre 2014 y 2065 proyectada por el INE (INE, 2014). El ejemplo considerado es válido cualquiera que sea la evolución futura del PIB, aunque es posible compensar la reducción de la población en edad de trabajar, de 31 a 21,2 millones entre 2014 y 2064 con un aumento de la productividad y del empleo, ya que el PIB puede crecer aunque disminuya la población adulta (Fernández Cordón y Planelles, (2014). En este caso, sería suficiente que el empleo pase del $56,5 \%$ actual de la población en edad de trabajar a 65\% en 2064 y que la productividad se incremente una media del $0,5 \%$ anual para que el PIB se mantenga constante hasta 2064. Este 0,5\% anual de productividad sería, en cierto modo, el "coste" de la evolución demográfica, adversa hasta aproximadamente 2050. La posibilidad de acudir a la inmigración y mayores tasas de incremento de la productividad permitirán, en el futuro, un crecimiento del PIB a tasas comparables a las observadas en las últimas décadas, si el sistema productivo funciona adecuadamente. 
El porcentaje del PIB que consumen los mayores depende por una parte del nivel de vida general, función este de la fracción del PIB que va a salarios $(\alpha)$, y de los ingresos de los mayores en relación con el resto de la población $(v)$. Por ejemplo, si las personas de 65 o más años reciben, en promedio, un $20 \%$ más que los de menos de 65 años y con un 50\% del PIB dedicado a remunerar el trabajo, los mayores se llevarían el 10,5\% del PIB en 2014 y el 21,6\% en 2064 (Cuadro 2 del Anexo técnico). Ese es el porcentaje que mantendría en 2064 la distribución actual de la renta, desde este punto de vista, equivalente al actual. No alcanzarlo significaría reducir el nivel de vida relativo de los mayores (y aumentar el de los más jóvenes) si $\alpha$ no varía, o reducir el nivel de vida del conjunto de la población si $\alpha$ disminuye. En el ejemplo se ha considerado que el consumo relativo de los mayores no aumenta en el futuro. Esto podría no verificarse si aumenta el coste de la dependencia, debido al aumento de la longevidad y al encarecimiento de la medicina, sobre todo si se confirma la tendencia a su privatización. No se debe por tanto descartar la hipótesis de que sea necesario un ajuste de la distribución de la renta que permita absorber este coste adicional. Sin embargo, la referencia, en tal caso, no sería el porcentaje actual del PIB que va a mayores sino el derivado de un cálculo como el nuestro, que tenga en cuenta el previsible cambio en la composición por edades de la población.

Para mantener constante en el futuro el consumo per cápita de todos los grupos de la población, es necesario que, además de que se mantenga el reparto primario entre capital y trabajo, la parte del PIB dedicada a mayores aumente cuando aumenta el peso de los mayores en la población y, consecuentemente, disminuye el peso de los más jóvenes. De no ser así, se producirá una redistribución de la riqueza creada que castiga exclusivamente al grupo de mayores, cuyos ingresos estarían entonces inversamente ligados a su importancia en la población, lo que no ocurre en los otros grupos.

La muy extendida advertencia de que, debido al envejecimiento demográfico, el porcentaje del PIB dedicado a pensiones (o en general a mayores) se puede disparar en el futuro hasta alcanzar niveles inasumibles por nuestra economía (se habla de 16 o $17 \%$ para pensiones, frente a los aproximadamente $9 \%$ de ahora) no se justifica en absoluto. Si el gasto dedicado a los mayores no sigue al aumento de su peso en la población, siempre que, al menos, se mantenga el nivel del PIB, se producirá una redistribución de riqueza, a priori injusta, a favor de los más jóvenes o de las rentas del capital.

El aumento de la productividad incrementa la riqueza producida por encima del crecimiento del empleo y, como ya se ha dicho, contribuye a compensar la caída de la población en edad de trabajar. Más allá, el crecimiento del PIB facilita sin duda la búsqueda de mecanismos que eviten una redistribución de la renta en detrimento de los mayores, pero el crecimiento no es una condición necesaria. Es difícil entender y admitir que en una economía capaz de producir más con menos gente, se considere inevitable reducir el nivel de vida de los mayores.

Por el contrario, si la disminución de la población en edad de trabajar llevara a una disminución del PIB, por falta de mano de obra o por insuficiencia de la 
productividad, nos encontraríamos ante un crecimiento negativo de la economía, con consecuencias no deseadas en muchos ámbitos de la vida social y económica, no solo el de las pensiones, y que no afectaría solo a los mayores. Parece, sin embargo, más verosímil que, en el futuro, un aumento de la tasa de empleo (sobre todo en España donde es especialmente baja) y de la productividad, completado eventualmente por la llegada de inmigrantes, permita cierto crecimiento económico, a pesar de que disminuya la población en edad de trabajar. La tendencia actual de producir cada vez más con menos empleo y menor salario plantea el grave problema de como canalizar la riqueza creada para atender las necesidades de la población. No hay duda de que los dispositivos de protección que están basados principalmente en la condición de asalariado y en el montante del salario, pueden entrar en crisis, no por falta de recursos, sino porque no funcionen los mecanismos ideados para otros momentos de la historia.

\section{Conclusión}

Un marco conceptual, como el que domina el análisis económico actual, que separa artificialmente ámbitos que en la realidad están estrechamente relacionados, no facilita la búsqueda de soluciones a muchos de los problemas actualmente planteados. Frente al supuesto peligro demográfico, considerado como una variable exógena, ajena a la economía, se encara el sistema de pensiones con un punto de vista estrechamente contable, aplicándole una lógica de contributividad estricta que no es la suya, sin replantear los canales de financiación. Un planteamiento que inevitablemente conduce a vaticinar la quiebra del sistema público, a pesar de que los recursos globales aumentan y de que existe la posibilidad de atender las necesidades de un mayor número de pensionistas sin atentar al nivel de vida medio del resto de la población. Decir esto no significa negar que exista un problema. Conduce, simplemente, a orientar la búsqueda de soluciones hacia los mecanismos que garanticen que los cambios demográficos se integran en la distribución de los recursos. En otras palabras, a indagar en los cambios, algunos sin duda muy profundos, que tienen que darse en nuestra manera de producir, de repartir y de consumir para adaptar nuestras vidas al nuevo modelo demográfico, que tantas ventajas tiene para la humanidad.

No es extraño que, frente a esta persistencia en el error, algunos invoquen la injerencia de los intereses de sectores a los que beneficia el declive del sistema público, para explicar los diagnósticos y las propuestas, todos conducentes a debilitar el sistema y a reducir las pensiones públicas. Tal vez haya que relacionar estas actitudes ante el tema de las pensiones, con los análisis y las propuestas que han conducido a imponer en la Unión Europea unas políticas de austeridad, que cada vez más analistas solventes consideran haber sido un error. Unas medidas que han provocado el estancamiento económico y el sufrimiento de millones de personas, y a las que, al parecer, tanto trabajo cuesta renunciar. El ámbito de las pensiones añade el carácter tan creíble del miedo al envejecimiento demográfico, 
debido, sin duda, a todas las connotaciones negativas que arrastra, aceptado de buena fe como amenaza real por muchas personas y muchos círculos, cuando se trata de un cambio social que simplemente exige adaptaciones que están a nuestro alcance.

\section{Anexo técnico}

Consideremos que el conjunto de las remuneraciones del trabajo representa una fracción $\alpha$ del PIB, $S_{t}=\alpha_{t} Y_{t}$, en el año t, formada por los salarios de los ocupados, que aseguran su propio consumo y el de todos los dependientes menores de 65 años, niños y adultos no ocupados, o sea la población de 0 a 65 años, y por los ingresos de los jubilados, como fuente del consumo del conjunto de las personas de 65 o más años. Se trata de una versión muy simplificada de la realidad, que nos permitirá sin embargo establecer relaciones claves para el análisis de las consecuencias de la evolución demográfica futura.

La renta del trabajo per cápita de la población: $\beta_{t}=\frac{\alpha_{t} Y_{t}}{P_{t}} \rightarrow \beta_{t}=\alpha_{t} \frac{Y_{t}}{P_{t}} \mathrm{y}$
$S_{t}=\beta_{t} P_{t}$

De la misma manera:

$S_{m, t}=\beta_{m, t} P_{65+, t} \quad$ ingresos de los mayores

$S_{r, t}=\beta_{r, t} P_{0-64, t}$ ingresos del resto de la población

Siendo $\beta_{m, t} \mathrm{y} \beta_{r, t}$, el ingreso medio del trabajo de los grupos correspondientes

Definimos ahora $v_{t}=\frac{\beta_{m, t}}{\beta}$ como el coeficiente de nivel de vida de los mayores con relación al resto de laßpoblación (personas de 0-64 años) en el año t. No habrá redistribución entre las edades si, en los años siguientes, $v$ se mantiene constante, cualquiera que sea el nivel del PIB y el valor de $\alpha$. Una disminución de $v$ implica un deterioro de la situación de los mayores y, al contrario, el aumento de $v$ supone que los mayores mejoran su situación relativa.

El ingreso total es:

$S_{t}=\beta_{t} P_{t}=\beta_{r, t} P_{0-64, t}+\beta_{m, t} P_{65+}=\alpha_{t} Y_{t}$

y sustituyendo

$$
S_{t}=\beta_{t} P_{t}=\frac{\beta_{m, t}}{v} P_{0-64, t}+\beta_{m, t} P_{65+}=\alpha_{t} Y_{t}
$$

y el ingreso per cápita del año t se expresa como:

$\alpha_{t} \frac{Y_{t}}{P_{t}}=\beta_{t}=\frac{\beta_{m, t}}{v} \frac{P_{0-64, t}}{P_{t}}+\beta_{m, t} \frac{P_{65+, t}}{P_{t}}$ 
Llamando $R M_{t}=\frac{P_{65+, t}}{P_{t}}$ proporción de mayores en la población total

$\alpha_{t} \frac{Y_{t}}{P}=\beta_{t} P_{t}=\frac{\beta_{m, t}}{t}\left(1-R M_{t}\right)+\beta_{m, t}(R M)_{t}$

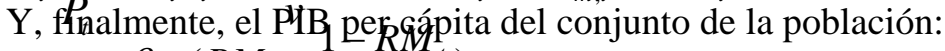

$\frac{Y_{t}}{R}=\frac{\beta_{m, t}\left(R M_{t}+\frac{1-R M_{t}}{v}\right)}{2}$

De $P_{0}$ anterior sW deriva la expresión del ingreso per cápita de los mayores:

$\frac{\beta_{m, t}}{\text { EPangreso total de-rquayores: }}=\frac{P_{t}}{P_{65+, t}+\frac{P_{0-64+, t}}{P_{t}}}$
$S_{m, t}=\frac{P_{65+, t}}{\beta_{m, t} P_{65+, t}}+\frac{Y_{V_{t}} P_{65+, t} \alpha_{t}}{P_{0-64, t}}$

La proporción del PIB ${ }^{65}$ detdicadara mayores es ahora:

$$
\delta_{m, t}=\frac{S_{m, t}}{Y_{t}}=\frac{P_{65+, t} \alpha_{t}}{P_{65+, t}+\frac{P_{0-64+, t}}{v_{t}}}=\frac{\alpha_{t}}{1+\frac{P_{0-64+, t}}{v_{t} P_{65+, t}}}=\frac{v_{t} \alpha_{t}}{v_{t}+\frac{P_{0-64+, t}}{P_{65+, t}}}=\frac{v_{t} \alpha_{t}}{v_{t}+\frac{1-R M_{t}}{R M_{t}}}
$$

estando formulada en términos de $\alpha$, porcentaje del PIB que remunera el trabajo, $v$, nivel de vida relativo de los mayores en relación al resto de la población y una expresión basada en la proporción de mayores en la población, $R M$.

Si mantenemos constante en el tiempo la distribución del PIB entre factores y la de los ingresos del trabajo entre los mayores y el resto de la población, la evolución de la proporción del PIB que va a mayores aumenta cuando aumenta la proporción de mayores.

La fórmula anterior se ha aplicado a la evolución de la proporción de 65 o más años de la población de 2014 a 2064, según las proyecciones más recientes del INE (INE, 2014) y atribuyendo unos valores numéricos, solo a título de ejemplo, a las dos variables restantes (Cuadro 2). Se ha considerado, para el nivel de vida de los mayores, un valor de 0,8 (menor ingreso per cápita que el resto) y de 1,2 (mayor ingreso per cápita que el resto) y para el coeficiente $\alpha$ tres valores: $40 \%, 50 \%$ y $60 \%$. Este ejemplo no tiene significado en sí: solo interesa verificar la evolución del porcentaje del PIB dedicado a mayores cuando aumenta fuertemente la proporción de 65 o más años (de 18,2\% en 2014 a 38,7\% en 2064, según las proyecciones del INE de 2014) y permanece constante la distribución relativa de la renta. No se introduce aquí un posible crecimiento del PIB, que podría facilitar la redistribución de la renta (por ejemplo a favor de los mayores) sin merma del nivel de vida del resto o con incremento desigual del nivel de vida de cada grupo. 
Cuadro 2 Porcentaje del PIB a mayores, según parámetros de distribución de la renta y proyección de población INE 2014 (ejemplo numérico)

\begin{tabular}{|r|r|r|r|r|}
\hline \multirow{2}{*}{$\begin{array}{c}\text { Ingresos } \\
\text { del trabajo }\end{array}$} & \multicolumn{3}{|c|}{$\begin{array}{r}\text { ingreso relativo per cápita de la población de 65+ } \\
\text { con relación a la población de 0-64 años }\end{array}$} \\
\cline { 2 - 5 } como & \multicolumn{2}{|c|}{0,8} & \multicolumn{2}{|c|}{1,2} \\
\cline { 2 - 5 } del PIB & 2014 & 2064 & 2014 & 2064 \\
\hline $40 \%$ & $6,0 \%$ & $13,4 \%$ & $8,4 \%$ & $17,3 \%$ \\
$50 \%$ & $7,5 \%$ & $16,8 \%$ & $10,5 \%$ & $21,6 \%$ \\
$60 \%$ & $10,5 \%$ & $20,1 \%$ & $12,6 \%$ & $25,9 \%$ \\
\hline
\end{tabular}

Fuente: elaboración propia con proyecciones de población INE 2014

\section{Bibliografía}

Bloom, D. E., Canning, D. y Sevilla, J, (2003). The demographic dividend: a new perspective on the economic consequences of population change. RAND Monographs Reports MR1274. http://www.rand.org/pubs/monograph_reports/MR1274.html

Castel, R. (1995). Les métamorphoses de la question sociale. Paris : Fayard.

Charpentier, F. (2009). Les retraites en France et dans le monde. Paris: Ed. Economica.

Conde-Ruiz, J.I. (2014). ¿Qué será de mi pensión? Cómo hacer sostenible nuestro futuro como jubilados. Madrid: Península.

Donzelot, J. (2005). La police des familles. Paris: Les éditions de Minuit.

Etxezarreta, M. (2009). La tendencia a la privatización: consideración especial de la privatización de las pensiones. En M. Etxezarreta et al., ¿¿Qué pensiones, qué futuro? El Estado de bienestar en el siglo XXI (pp 9-80). Barcelona: Icaria.

Fernández Cordón, J.A. y Planelles Romero, J. (2014) Integrating labor market in population projections. En Proceedings of the Sixth Eurostat/Unece Work Session on Demographic Projections. Roma: ISTAT (pp 299-312) 
Guillemard, A.M. (2007). Une nouvelle solidarité entre les âges et les générations dans une société de longévité. En Paugam S. Repenser la solidarité (pp 355375). Paris PUF.

HMDB (2015). Human Mortality Database. Spain: Mortality Tables 1x1. http://www.mortality.org/cgi-bin/hmd/country.php?cntr=ESP\&level=1 consultado 12/2/2015.

IESS: International Encyclopedia of the Social Sciences. 2008. "Reciprocity, Norm of." Encyclopedia.com. 7 Mar. 2015 http://www.encyclopedia.com

INE (2014). Proyecciones demográficas. Parámetros de evolución demográfica. http://www.ine.es/inebaseDYN/propob30278/propob_resultados.htm

Jimeno, J.F. y Licandro O. (1999). La tasa interna de rentabilidad y el equilibrio financiero del sistema español de pensiones de jubilación. Investigaciones Económicas, vol XXIII (1), 129-143.

Kotlikoff, L.J. (2004). Pensions systems and the intergenerational distribution of resources. Working Paper 36/04. Center for Research on Pensions and Welfare Policies

Kotlikoff, L.J. (2006). Paul Samuelson's Amazing intergenerational Transfer. En Szenberg M., Ramrattan L. y Gottesman A. (eds). Samuelsonian Economics and the twenty-first century (pp 42-53). Oxford University Press.

London, J. (1901). The law of life (The children of frost). En London, J. (2014). Complete Works of Jack London (illustrated). Delphi Classics.

Mason A. (2006). ¿Cuál es el dividendo demográfico? Finanzas \& Desarrollo, Washington, FMI, septiembre: 16-17.

Ostner, I. (2004). What are Children for?: Reciprocity and Solidarity between Parents and Children. En Knijn, T. and Komter A. (eds.). Solidarity Between the Sexes and the Generations (pp 167-184). Cheltenham: Edward Elgar.

Samuelson, P.A. (1958). An Exact Consumption-Loan Model of Interest with or without the Social Contrivance of Money. Journal of Political Economy, Vol. 66, Núm. 6, 467-482.

Shkolnikov, V., Andreev E.M., Jdanov, D., Jasilionis, D., Kravdal, Ø, et al. (2012). Increasing absolute mortality disparities by education in Finland, Norway and Sweden, 1971-2000. Journal of Emidemiology and Community Health, 66(4): 372-378. 
Tobío Soler, C. (2012). Reciprocity and solidarity in intergenerational relationships: Spain, France and Norway in comparative perspective. Papers, 97/4, 849-873.

Tobío Soler, C y Fernández Cordón J.A. (2013). Family networks in Andalusia, Spain. International Review of Sociology, 23,1, 68-84.

Waldron, H. (2007). Trends in Mortality Differentials and Life Expectancy for Male Social Security-Covered Workers, by Socioeconomic Status. Social Security Bulletin, Vol. 67, Num. 3, 28 p. 